\title{
A RANDOMIZED TRIAL OF A MULTIMODAL COMMUNITY-BASED PRISONER REENTRY PROGRAM EMPHASIZING SUBSTANCE ABUSE TREATMENT
}

Eric Grommon, Ph.D.

Assistant Professor

School of Public and Environmental Affairs

Indiana University-Purdue University Indianapolis

801 W. Michigan Street, BS 4067

Indianapolis, IN 46202

317/278-9481

317/274-5153 (fax)

egrommon@iupui.edu

William Davidson, Ph.D.

University Distinguished Professor

Michigan State University

Department of Psychology

Michigan State University

132 Psychology Building

East Lansing, MI 48824

517/353-5015

517/432-2945 (fax)

davidso7@msu.edu

and

Timothy Bynum, Ph.D.

Professor

Michigan State University

School of Criminal Justice

560 Baker Hall

East Lansing, MI 48824

517/355-2196

517/432-1787 (fax)

bynum@msu.edu

KEYWORDS: Prisoner Reentry, Substance Abuse Treatment, Program Evaluation, Corrections, Parolees.

This is the author's manuscript of the article published in final edited form as:

Grommon, E., II, W. S. D., \& Bynum, T. S. (2013). A Randomized Trial of a Multimodal CommunityBased Prisoner Reentry Program Emphasizing Substance Abuse Treatment. Journal of Offender

Rehabilitation, 52(4), 287-309. http://doi.org/10.1080/10509674.2013.782775 


\title{
A RANDOMIZED TRIAL OF A MULTIMODAL COMMUNITY-BASED PRISONER REENTRY PROGRAM EMPHASIZING SUBSTANCE ABUSE TREATMENT
}

\author{
ABSTRACT \\ Prisoner reentry programs continue to be developed and implemented to ease the process \\ of transition into the community and to curtail fiscal pressures. This study describes and \\ provides relapse and recidivism outcome findings related to a randomized trial evaluating a \\ multimodal, community-based reentry program that prioritized substance abuse treatment. \\ Results from analyses suggest negligible effects for participants of the program across multiple \\ outcome indicators. Avenues for further exploration are provided, with a cautionary emphasis \\ on the continued expansion of multimodal community-based reentry programs.
}


Two-thirds of state prisoners have diagnosable drug addictions (National Center on Addiction and Substance Abuse, 2010). Most of those in need of substance abuse treatment will leave prison without any form of intervention (Mumola \& Karberg, 2006). Few will receive intense, prison-based therapeutic community substance abuse treatment despite its acceptance as an effective form of intervention to reduce relapse and recidivism (Mitchell, Wilson, \& MacKenzie, 2007; Pearson \& Lipton, 1999; Prendergast, 2009). The gap in access to effective treatment, which forms the critical foundation for continuity and aftercare services (Chandler, Fletcher, \& Volkow, 2009; Pelissier, Jones, \& Cadigan, 2007), has led to the development of broad, multimodal approaches to initiate community-based substance abuse treatment and provide transitional assistance.

Relative to evaluations of in-prison therapeutic communities, drug courts, and diversionary interventions, the knowledge base on standalone community-based substance abuse treatment for offenders is underdeveloped, mixed, and plagued by poor research designs (Chanhatasilpa, MacKenzie, \& Hickman, 2000; Hiller, Knight, Saum, \& Simpson, 2006; Perry, Darwin, Godfrey, McDougall, Lunn, Glanville, \& Coulton, 2009). Nevertheless, communitybased substance abuse treatment continues to be integrated into reentry program designs to cover a breadth of needs upon release. It remains to be determined if this approach is an optimal strategy. Results of recent multimodal, community-based program evaluations have shown promise with relapse and/or recidivism reductions (Duwe, 2012; Jacobs \& Western, 2007; Lattimore \& Visher, 2010; Sample \& Spohn, 2008; Zhang et al., 2006) or questioned the utility of such programs due to null findings or increased rates of relapse and/or recidivism (Bloom, Redcross, Zweig, \& Azurdia, 2007; Bouffard \& Bergeron, 2007; McDonald, Dyous, \& Carlson, 2008; Lattimore et al., 2012; Minnesota Department of Corrections, 2011; Prendergast, Frisman, 
Sacks, Stanton-Tindall, Greenwood, Lin, \& Cartier, 2011; Redcross, Bloom, Azurdia, Zweig, \& Pindus, 2009; Rossman \& Roman, 2003;; Severson, Bruns, Veeh, \& Lee, 2011; Smith \& Suttle, 2008; Wilson \& Davis, 2006).

The purpose of the study reported here was to evaluate the effectiveness of a multimodal, community-based prisoner reentry program that emphasized substance abuse treatment for offenders with substance dependencies. Informed by treatment theory and best practice literatures, the program focused on a high-risk sample and attempted to meet participant need. Treatment strategies purported to maximize learning, increase motivation, and cater to participant strengths were central to program design and operation (Andrews et al., 1990; Cullen \& Gendreau, 2000; Lowenkamp, Latessa, \& Smith, 2006). A cognitive-behavioral therapeutic framework was used to increase the likelihood for behavioral change. This approach fundamentally assumed that substance dependence is a product of learned behavior that can be unlearned and/or replaced (Anglin \& Hser, 1990) and is considered to be one of the most promising techniques in the correctional field (Andrews et al., 1990; Cullen \& Gendreau, 2000; Landenberger \& Lipsey, 2006; Lipsey, Chapman, \& Landenberger, 2001; MacKenzie, 2006). In addition to these core principles, the multimodal approach took place over an extended period of time to foster and reinforce behavioral change (Cullen \& Gendreau, 2000). Drug testing was also used to condition behavior and hold participants accountable (Fletcher \& Chandler, 2006).

Two focal concerns were addressed in this study. First, did this program reduce relapse? Second, did this program reduce recidivism?

\section{METHODS}

Research Design 
An intent-to-treat experimental design with random assignment of eligible offenders to the program participant (treatment) group and traditional community supervision group (control) was employed. A priori power analyses were conducted to determine statistical power and specify the sample size (Cohen, 1988; Erdfelder, Faul, \& Buchner, 1996). Meta-analyses of community-based substance abuse treatment for correctional populations guided anticipated effect size estimates (Aos, Miller, and Drake, 2006; Prendergast, Podus, Chang, \& Urada, 2002). To identify a 10\% decline in the outcome measures with conventional statistical power (.80) and Type I error (.05) a total sample size of 390 individuals was required. The design included a 2 year post-release follow-up of approximately 500 offenders (250 per condition) who screened eligible to the program.

\section{Participants}

The program was designed to reach a target population of at-risk male offenders soon to be released from prison, placed under 24 month community correctional supervision, with significant substance dependencies. During a two and a half year period, all male individuals scheduled to be released to the study county were screened for eligibility. Parole agents conducted the eligibility screens using a standardized protocol. Parole supervisors were responsible for the review and confirmation of eligibility determinations.

Substance dependency was identified by a combination of screening inventory, criminal history, and case file review through the pre-parole process. Assessment of substance dependence was achieved through the use of the Substance Abuse Subtle Screening Inventory (SASSI) (Lazowski, Miller, Boye, \& Miller, 1998; Miller \& Lazowski, 1999). SASSI was the standard inventory used by the Department of Corrections to determine and forecast treatment needs of correctional populations. Only offenders with high probability of substance dependence 
or severe dependence were eligible. Risk determinations were made with the use of the Department of Corrections' internal variation of salient factor scores. These assessments are largely driven by criminal history, conviction offense information and institutional misconduct (Hoffman \& Beck, 1974). High and medium risk offenders were eligible for inclusion into the program.

Exclusionary criteria also eliminated those with high assaultive risk classifications that prohibit placements to community services, sex offense histories, arson histories, pending new felony charges, physical or mental conditions that may prohibit participation, paroles from other states under interstate compacts, and those assigned to minimum community correctional supervision status from the eligibility pool. ${ }^{1}$ The latter criterion was used to exclude low-risk offenders.

If an individual was determined to be eligible, they were entered into a lottery for random assignment to program or control group conditions. Assignment to conditions was involuntary in the sense that all eligible individuals were referred and required to enter substance abuse treatment as a condition of community supervision. Institutional Review Board approval was obtained for the program evaluation.

A total of 511 eligible male offenders paroled during the study interval. Of those paroled, 263 were randomly assigned to the Treatment Group and the remaining 248 to the Control Group. Straight random assignment procedures were used without blocking or stratification variables. The parole board and parole agents were blind to the random assignment procedures. Table 1 displays the demographic characteristics of the two groups [Insert Table $\mathbf{1}$ about here]. Two-way analysis of variance (ANOVA) and chi-square models were used to assess differences between the conditions. Even though randomly assigned, the Treatment Group was significantly 
older and a higher proportion of the Control Group were high school graduates. No other demographic comparisons yielded significant differences between the groups. With one exception, there were no significant differences on any of the criminal or substance dependence histories. The Control Group was significantly more likely to have participated in substance abuse treatment one year prior to parole. ${ }^{2}$

Procedures

Measures

Data were compiled from multiple sources over the course of the 2 year post-release follow-up period. Management information systems of the Department of Corrections and State Police were used to obtain relevant demographic and background information. Re-arrest measures were extracted from State Police management information systems. Relapse and reincarceration measures were derived from the Department of Corrections management information system extractions. Databases from drug treatment and service providers were used to measure program participation and compliance. Two local non-profit providers were contracted through a competitive proposal process to provide treatment and services to program participants.

Independent Variables

All participants were involved in one of two experimental conditions. The Treatment Group was exposed to a specific treatment intervention for the first year following prison release, while the Control Group was exposed to standard parole conditions. All participants, regardless of condition, were subjected to the standard conditions of parole during the subsequent twelve month follow-up interval. 
Treatment Group. The structure and content of the treatment program included graduated transitioning in two phases over the course of the first year after release. Phase One took place during the first 30 to 45 days post-release and required residence in a secure transitional facility. This phase focused on establishing housing placement, obtaining employment, enrolling in employability and life skills training, and starting outpatient substance abuse treatment services. Contact with family members was also made in order to assess need for family-based substance abuse treatment and initiate family sessions as necessary.

Treatment protocols followed a blend of standardized cognitive behavioral strategies for self-improvement and change (Wanberg \& Milkman, 1998) and a social learning based motivational interviewing curriculum (Miller \& Rollnick, 2002; Tomlin \& Richardson, 2004). Protocols were manualized for therapist use and participants received workbooks associated with the curriculum. Sessions were also supplemented by 12-step facilitation therapeutic techniques (Stinchfield \& Owen, 1998). Therapists were trained in cognitive-behavior therapy principles and possessed years of behavioral recognition and relapse prevention experience with community-correctional populations. All therapists had baccalaureate degrees, were certified, and licensed addiction counselors. Staff received training in evidence based practice principles from external, accredited organizations on a semi-annual basis and quarterly internal in-service workshops from supervisors.

Participants were managed under a specialized community supervision caseload at release. A primary caseworker, treatment therapist, and case coordinator were assigned upon intake to the program. The caseworker assessed participant need (i.e., educational, employment, housing, personal identification, mental/physical health, health care, transportation, and clothing) and the primary therapist clinically assessed substance dependence history and designed 
individualized treatment protocols. This team then developed treatment and relapse prevention plans and referred participants to organizations in the community who could assist with specified needs. Program staff worked collaboratively to identify goals and objectives for participants and monitor participant progress on a weekly basis. After two weeks in Phase One, a treatment plan review was held with program staff, the participant, and the participant's community correctional supervisory agent. This review determined if initial goals and objectives were being met and whether to extend the Phase One or allow transition into Phase Two.

Substance abuse treatment during Phase One consisted of 10 hours of direct services per week. These ten hours included 1 individual counseling session (1 hour), 3 group/didactic sessions (4.5 hours), 1 family group therapy session (1.5 hours), and 3 Alcoholics Anonymous or Narcotics Anonymous meetings (3 hours). Drug testing in Phase One was conducted each day of the week. A randomization protocol was used to generate daily testing numbers and assure that $40 \%$ of the participants were tested at least twice per week. If the participant's assigned testing number was called, the participant was required to submit a drug test at the testing wing of the transitional facility. Non-compliance with treatment participation and positive drug tests resulted in graduated programmatic sanctions.

Transition to Phase Two allowed the participant to move to an approved home residence and was contingent upon compliance with substance abuse treatment and testing. Phase Two required continued participation in individual and family substance abuse treatment through the remainder of the first post-release year. The primary therapist and case coordinator continued to monitor and assist in the obtainment of program goals and objectives. Bi-monthly and monthly meetings were held to review progress. Participants graduated from Phase Two after meeting substance abuse treatment objectives and demonstrating sustained behavioral stability. Program 
completion determinations were made by the primary therapist, case coordinator, and community supervisory agent. Participants could only be removed from the program through graduation or the termination of parole supervision.

During Phase Two, substance abuse treatment consisted of two group/didactic sessions (3 hours), one family group session (1.5 hours), and three Alcoholics Anonymous or Narcotics Anonymous meetings (3 hours) per week. Participants also received two individual counseling sessions per month. Randomized drug testing in Phase Two was conducted each business day. Participants in Phase Two were required to call a toll-free phone number each day to check the listing of testing numbers. If the participant's assigned testing number was called, the participant reported to the transitional housing facility for testing. Once again, the randomization protocol used to generate daily testing numbers assured that $40 \%$ of the participants were tested at least once each week. Positive drug tests resulted in immediate three day sanctions to be served at the transitional housing facility. Continued non-compliance with treatment or testing led to additional three day sanctions or graduated sanctions.

One distinguishing component of this program was in-reach by program staff. Once a participant had been identified, contact with the case coordinator initiated and the process of planning substance abuse treatment services and coordinating referrals to community agencies and the obtainment of necessary documents began. This activity occurred before the participant was released.

Control Group. The control group was directly released according to traditional prerelease and community supervision plans. As is the case in traditional parole for those who screen with substance dependency problems, release conditions mandated referral to substance abuse treatment services. This group could participate in a variety of ad hoc substance abuse 
treatment services in the community. They were subjected to drug testing and violations for noncompliance with testing procedures and were monitored with an equivalent community supervision schedule to the participant group. Beyond these similarities, the control group did not receive pre-release program in-reach and subsequent program services, did not participate in the transitional housing component of the program, and did not receive intensive case management oversight for the first twelve months post-release that is provided to the participant group.

Manipulation Checks

To assess the implementation of the experimental conditions, a variety of variables were examined. Program fidelity variables were collected for members of the Treatment Group across their length of stay in the program. These variables were used as a metric of program participation. The remaining manipulation check variables were collected across the two-year follow-up period to observe comparability across the experimental conditions.

Program Fidelity to Participation Thresholds. Substance abuse treatment was central to the program design. To determine levels of participation for the Treatment Group weekly count data were collected to observe variation in participation. Individual Counseling Sessions was defined as the average number of individual substance abuse counseling sessions attended by treatment group participants on a weekly basis, while Didactic Sessions was the average number of group substance abuse treatment therapy sessions. Family Group Sessions were the average number of family-based group substance abuse treatment therapy sessions and $A A / N A$ Support Group Sessions captures the average number of Alcoholics Anonymous or Narcotics Anonymous support group sessions treatment group members attended. For each of these measures a secondary indicator was used to identify the proportion of participants who attended 
at least one of the various treatment sessions. Finally, length of stay was defined as the average number of days participants remained in the program.

Frequency of Drug Tests. Frequency of tests was defined as the number of drug tests administered to participants. This measure was used to observe variation in testing between the experimental conditions across the study follow-up period.

\section{Dependent Variables}

All dependent variables were collected across a two-year period following prison release. The first year corresponded to participant behavior while exposed to differential conditions. The second year represents a release from conditions and return to standard parole for both groups.

Relapse. Relapse was primarily defined as the presence of any positive drug test over the course of the evaluation period. This dichotomous indicator of relapse was supplemented by a continuous measure of the percentage of positive tests, given the high likelihood of relapse among dependent populations (Anglin \& Hser, 1990). The secondary measure is a product of the ratio of positive tests to total tests administered. Urine screens were required as a condition of community supervision and were monitored by field agents. Panel urine screens were done for the following substances: heroin, cocaine, methamphetamine, marijuana. Measures of relapse do not count missed, adulterated, or other non-compliance with drug testing as a positive test.

Rearrest. Rearrest was defined as the arrest for a new felony offense. A focus on felony arrests is common in recidivism research since there is greater reliability in the report and documentation of such offenses relative to misdemeanors (Maltz, 1984; Travis \& Visher, 2005).

Reincarceration. Reincarceration was defined in two ways. The first was a Technical Violation. Community supervision could have been revoked for a violation of supervision conditions, which led to a prison return on the same sentence. The second was a New Offense. 
This type of reincarceration occurred through a conviction on a new sentence. Alternative measures of recidivism are used since a single measure can be influenced by policy or operational issues (Travis \& Visher, 2005).

\section{RESULTS}

By design, the Treatment Group was exposed to an intensive substance abuse treatment program. To ensure that this objective was met, program fidelity measures were used to determine average levels of exposure and weekly participation. Descriptive summary statistics are presented in Table 2. [Insert Table 2 here]

Over $90 \%$ of Treatment Group participants were exposed to some form of treatment services in Phase One. Substance abuse treatment was designed to consist of 10 hours of services per week in this phase. The rate of participation observed was comparable to program intentions but lower than anticipated. Participants received two fewer didactic sessions and one less support group session per week, which reduced the average to 6.5 hours of direct services. In Phase Two, exposure to didactic and individual counseling sessions remained high, but the rate of participation was lower than the program model. On average, participants attended one individual counseling session a month. The number of didactic sessions attended was considerably lower than the program model objectives. Participation in support group and family group sessions dramatically declined in Phase Two. The average length of stay was 300 days, which is slightly less than one post-release year and was highly variable between participants (range $=7-582 ; S D=112.79)$.

It was anticipated that the Treatment Group would be drug tested at a similar rate to the Control Group. To determine the degree of variability between the experimental conditions, the frequency of drug testing was examined. A two-way ANOVA model was used to observe 
differences in means between the conditions. The results indicate that members of the Treatment Group were tested at a significantly higher rate than the Control Group $[F(1,509)=50.79, \mathrm{p}<.01$, partial-eta squared=.09]. The frequency of testing was twice as high for the Treatment Group $\left(M_{t}\right.$ $\left.=81, S E_{t}=4.55 ; M_{c}=34, S E_{c}=4.68\right)$.

The results highlight a number of implementation concerns. First, the fidelity of the program was lower than anticipated and appeared to drop considerably over time. There is substantial exposure to treatment as indicated by the proportion of Treatment Group participants who attended at least one session. However, the levels of weekly participation were substantially low. Second, the equivalence in testing schedules between the conditions was not achieved. Instead, the Control Group was tested at a rate of 2 tests per month, which corresponds to the standard drug testing policy of the study state.

Relapse

Two primary statistical techniques were used to assess the effect of the program on relapse and recidivism outcomes. ANOVA models were used to determine the significance of differences between experimental conditions. ANOVA is an efficient method for the analysis of experimental design data (see Shadish, Cook, \& Campbell, 2002) and is robust when dichotomous outcomes are examined (D’Agostino, 1971; Lunney, 1970). Two-way main effects ANOVAs were estimated.

Survival analysis was also used to examine differences across the two-year follow-up relative to the timing of relapse and recidivism events. Results identified the proportion of the sample that did (failed) or did not (survived) incur the outcome of interest and the number of days until failure. Kaplan-Meier (1958) survival estimates allowed for the observation of statistically significant differences between groups in terms of the probability of and timing to 
relapse and recidivism events. The estimates were stratified by experimental conditions (Yamaguchi, 1991).

A majority of the overall sample relapsed at some point during the follow-up period. Table 3 provides a statistical summary of the results. There were no statistically significant differences between the two conditions in terms of drug use relapse. In comparing the two groups, $71 \%$ of the Control Group and 75\% of the Treatment Group relapsed. When the proportional measure of relapse was examined, significant differences between the two conditions were observed. On average, members of the Treatment Group tested positive in 26\% of their drug tests, while $21 \%$ of the tests administered to the Control Group resulted in a positive test. [Insert Table 3 about here]

Time to relapse also differed between the two conditions. The mean survival time to relapse was 286 days for the Treatment Group and 338 days for the Control Group. The 2 to 3 month difference between the two groups was statistically significant, which confirms the baseline observation that Treatment Group relapse would be detected faster than the control group. Wilcoxon and Tarone-Ware test statistics include weights for initial time intervals and early relapses (Hosmer, Lemeshow, \& May, 2008). The statistics suggested that the differences between groups occur relatively soon after release and begin to converge over time, but remain statistically dependable throughout the follow-up period (see Figure A). [Insert Figure A about

\section{here]}

\section{Recidivism}

Most of the sample remained out of the criminal justice system during the follow-up period. Thirty percent (30\%) of the Treatment Group was rearrested and 36\% were returned to prison. Twenty-seven percent (27\%) of the Control Group were rearrested and 31\% were 
returned to prison. The Treatment Group appeared to be more likely to recidivate and recidivated faster than the Control Group. However, these trends and between-group differences were not statistically significant. Similar non-significant findings were observed when reincarceration was disaggregated into technical violation reincarceration or new sentence reincarceration. The differences between groups on reincarceration appear to be a function of technical violations as the rate and timing of return to prison for new sentences were nearly identical between the two groups. [Insert Figure B about here] DISCUSSION AND CONCLUSION

These results provide some rather discouraging findings concerning the effectiveness of a multimodal, community-based prisoner reentry program that included a substance treatment component conceived to be strong and informed by best practice. These results are particularly disheartening given that the program included active transitional elements, cognitive-behavioral components, and a relatively intense and lengthy intervention period. Further, the program was individually tailored based upon assessments of need. There was no evidence of effectiveness in terms of recurring substance abuse or multiple indicators of recidivism. Any trends observed indicated that the program increased relapse and recidivism.

Given these findings, a number of concerns arise. Overall treatment strength may not have been of sufficient consistency to impact outcomes. As is the case with many communitybased programs, dosage levels were highly variable between participants. To assess the effect of participation variability, weekly participation in primary substance abuse treatment services were aggregated across program length of stay to create a binary dosage proxy within the Treatment Group. This measure was based upon a median split of services received and separated individuals who received a high dose of treatment from those who received a low dose of 
treatment. With the use of the proxy measure and the verification of Control Group exposure to some form of substance abuse treatment, a three-level comparison between the Treatment Group by high and low dosage level and the Control Group was conducted. ${ }^{3}$

Two-way ANOVAs were estimated. There were no differences between program participation variability and the Control Group on the likelihood of relapse, rearrest, or reincarceration. Similarly, there were no differences between groups with regard to the proportion of positive tests, reincarceration on a technical offense, or reincarceration on a new offense. Variability in program participation did not appear to influence relapse or recidivism.

Participation variability was further examined within the Treatment Group. ANOVA analyses suggested that participants received equitable levels of substance abuse treatment in the first phase of the program. In other words, high dosage participants did not receive significantly more systematic intervention during Phase One that low dosage participants. Differential participation occurred in Phase Two. Participants who received a high dose of treatment participated in significantly more individual counseling $[F(1,261)=3.64, \mathrm{p}<.05]$ and group/didactic $[F(1,261)=16.21, \mathrm{p}<.001]$ substance abuse treatment sessions than those who received a low dose.

It appears that slightly higher levels of participation in individual counseling and group/didactic sessions over a longer period of time separated high and low levels of participation. In turn, the higher dosages received influenced recidivism outcomes for members of the Treatment Group, but dosage levels were not associated with relapse outcomes. Participants who received a high dose of treatment were significantly less likely to be rearrested $[F(1,261)=3.52, \mathrm{p}<.001]$, reincarcerated in general $[F(1,261)=71.93, \mathrm{p}<.001]$, or 
renincarcerated on a technical violation $[F(1,261)=27.41, \mathrm{p}<.001]$ or new sentence $[F(1$, 261) $=21.29, \mathrm{p}<.001]$.

This suggests that if dosage levels were maintained throughout the program for a higher proportion of participants, the overall findings may have lent themselves towards more favorable outcomes. It is equally possible that these post hoc analyses identified the more compliant and “ready to change” participants (see Bushway \& Apel, 2012; Sung, Mahoney, \& Mellow, 2010). By self-selection, these participants may possess other unobservable individual or individualcontextual interaction characteristics that predict more desirable outcomes. ${ }^{4}$ However, as the supplemental results suggest, the intensity of treatment was lacking for a majority of participants and deteriorated with progression across the second phase of the program. This presents one of the long standing dilemmas of systematic intervention with offenders that the more intense the intervention in an open, community setting the more difficult program completion may become for the recalcitrant individual (Marlowe, 2011; Prendergast, Greenwell, Cartier, Sacks, Frisman, Rodis, \& Havens, 2009; Rossi, Lipsey, \& Freeman, 2004; Vaughn, Sarrazin, Saleh, Huber, \& Hall, 2002).

The findings from this study suggest that a multimodal reentry program approach emphasizing substance abuse treatment in unison with transitional housing, case management oversight, and exposure to established referral networks in the community does not appear to be the optimal strategy for offenders with substance dependencies. Beyond fidelity concerns, the findings should be considered with a methodological limitation in mind. Recall, members of the Control Group did not receive program services afforded to the Treatment Group. In accordance with standard parole practice, Control Group members were referred to substance abuse treatment services and could have participated in a variety of treatment services. Indeed it would 
be unethical and unfeasible to randomly assign participants to a condition that withholds treatment. Treatment migration is a common threat to the internal validity of field evaluations (Goldkamp, 2008). This threat cannot be entirely ruled out from this research. The fact that the Control Group received substance abuse treatment could partially explain why there were no differential effects between the experimental conditions.

With the benefit of hindsight, there may be speculative factors that account for why this program did not achieve its anticipated outcomes. In offering these insights, it must be made clear that future research is needed to test and substantiate claims. It remains to be seen if this type of program, implemented with a high degree of fidelity, would affect outcomes. Small to moderate average effect sizes are common amongst cognitive-behavioral approaches (Landenberger \& Lipsey, 2006). Despite best intentions to integrate and deliver cognitivebehavioral treatment protocols thought to affect substance use and criminal behaviors, the primary interventions used in this program may have been too weak for an at-risk population of individuals with extensive histories of substance dependence. This population is difficult to manage and provide services; many will be resistant to treatment given past experiences with a variety of interventions that may or may not have aided recovery (Dennis, Scott, Funk, \& Foss, 2005; Hser, Anglin, Grella, Longshore, \& Prendergast, 1997; Malik-Kane \& Visher, 2008). In short, it is important to remain realistic. Interventions with high-risk populations will provide the most room for gain in the reduction of problem behavior, but they will not be easy and require a strong degree of commitment among program stakeholders.

More problematic may have been the structure of service delivery during the immediate period of release and transition. Continuity of care was lacking as most participants received low-intensity substance abuse treatment services in prison. Few had received these services more 
than a year before their release. Upon transition, participants were required to start a relatively active and intense treatment program and reside in a secure transitional facility. The effect of this process on participant motivation and subsequent engagement in treatment is not clear. It is plausible initial experiences affected the agency of participants and their perceptions of procedural justice, which influenced participation and subsequent outcomes. For instance, Kras (forthcoming) noted a complicated mix of perceptions among 36 males subject to mandated treatment while under community supervision. Members of the sample oscillated between themes of resistance and acceptance, apathy and motivation, progressions and hindrances, and injustice and legitimacy that can change over time but leave lasting impressions. Continued exploration into the interplay and interrelationships between motivation, engagement, and procedural justice would make for productive contributions to the literature on substance abuse treatment and correctional intervention.

It may be the case that multimodal community-based reentry programs can only influence social capital or additional higher-order concepts that are unrelated to outcome indicators. For instance, Lattimore and colleagues (2012) found that substance abuse treatment was not associated with relapse or recidivism, but did reduce difficulties in obtaining housing. Alternatively, the capacity of substance abuse treatment to affect behavior may be diluted when packaged within a multimodal reentry program. Grommon (2013) observed that housing and employment difficulties affected levels of participation in community-based substance abuse treatment. The ability to obtain basic needs often overshadow substance abuse treatment needs (Morani et al., 2011). It is not possible to dissect the program components examined here. At the molar level a specific model of substance abuse treatment is entangled with community transition and reentry strategies. To the extent that these approaches may have worked at cross 
purposes or been confounded in other ways, the results become difficult to interpret directly. However, it needs to be pointed out that this is a common issue when evaluating most interventions and the intervention model examined here combined elements that all have theoretical support for reduction of drug use and recidivism.

Among substance abuse treatment interventions, therapeutic communities, drug courts, and cognitive-behavioral approaches have provided sustained levels of effectiveness, especially when an aftercare component is included (Bahr, Masters, \& Taylor, 2012). The most promising multimodal, community-based reentry approaches are those that are coordinated by institutional and field correctional staff to ensure continuity through case management (Duwe, 2012; Olson \& Rozhon, 2011; Sample \& Spohn, 2008; Zhang et al., 2006), at minimum emphasize outpatient substance abuse treatment modalities (Jacobs \& Western, 2007; Zhang et al., 2006), and can incorporate transitional housing and/or employment placements (Bloom et al., 2007; Jacobs \& Western, 2007; Redcross et al., 2009). These components establish a minimum baseline of assistance and intervention. However, there are many overlaps in the evidence between community-based substance abuse treatment interventions and reentry programs that show and fail to show effects. Much of this evidence is shaped by differing levels of system coordination, program implementation, target populations, and methodological rigor. At this time, additional quality research is necessary to refine avenues for program structure and content that leads to reductions in relevant outcomes.

This study adds to the growing body of evidence that new correctional programs need to be scrutinized for their effectiveness in scientifically valid ways. The key ingredients for future work will include several factors. First, the use of scientifically credible research designs is critical. These issues are simply too theoretically and socially important to rely on non- 
experimental designs. Second, future research needs to make every effort to examine high intensity interventions which are consistently delivered to all individuals. While the program examined here had some of these elements, the degree of variability in program implementation, particularly during Phase Two, highlights this issue. Finally, future research can produce more lucid results by unpacking complex community-based interventions in a relative efficacy design. Only through the greater precision such approaches can add will the knowledge base, and hence utility, of these approaches be enhanced. 


\section{ENDNOTES}

1. Throughout the eligibility determination and random assignment process 1,094 individuals were deemed to be ineligible for program participation. Most (44\%) of the ineligible determinations were due to assessment as low-risk, followed by mental or physical health conditions $(13 \%)$ or a combination of these categories.

2. Developed from literature on therapeutic communities (Hiller, Knight, \& Simpson, 1999;

Wexler, Falkin, \& Lipton, 1990), continuity of care is a critical dimension of substance abuse treatment for correctional populations (Chandler, Fletcher, \& Volkow, 2009; Pelissier et al., 2007). This measure examines the timing of substance abuse treatment prior to release. All of the participants had received substance abuse treatment while in prison. Consistent with national trends (Mumola \& Karberg, 2006), treatment generally consisted of low-intensity services such as self-help groups or education-based programming.

3. Given that the Control Group served as a "standard procedure" counterfactual for the Treatment Group and substance abuse treatment data were only collected for participants of the intervention described here (i.e., the Treatment Group), there was concern about the comparability of the two groups. The issue focused on whether members of the Control Group were participating in substance abuse treatment. Given screening inventories which suggest this group was in need of treatment, observations of their participation would add confidence to the equivalence of participants randomly assigned to experimental conditions, but also raise concerns about the comparability of the experimental conditions. A supplemental data request was processed by the study state Department of Corrections to verify Control Group participation in community-based substance abuse treatment. It was determined that $70 \%$ of the Control Group had initially enrolled in outpatient substance abuse treatment. Most only received 
outpatient treatment services during their period of observation in this study. Thirty-four percent of those enrolled in treatment were placed into residential services after initial outpatient services. These data were obtained through a review of agent case note and billing information. Through discussions with the Department of Corrections it was anecdotally assumed that the remaining proportion of the Control Group had been at least referred to community-based substance abuse treatment. It is highly probable that these individuals were enrolled in treatment, but requisite information had not been entered by or forwarded to the Department of Corrections at the time of the data request.

4. The experimental design used in this study largely controlled for pre-existing differences between individuals, which allows for the determination of program effects. Uncontrolled individual differences do have the potential to influence outcomes. As a sensitivity check on the between-group differences observed in this study (see Table 1), supplemental ANOVAs were estimated. Overall, the observed background differences did not interact with group assignment to affect program outcomes. The only exception came from analyses of exposure to substance abuse treatment one year prior to release on reincarceration $[F(1,507)=7.23, \mathrm{p}<.01]$. Recall this measure was a proxy indicator of treatment continuity. Pairwise comparisons suggested that Treatment Group participants who received service continuity were significantly more likely to be returned to prison relative to Control Group participants who had similar continuity experiences. Reincarceration on a new sentence appeared to be the mechanism influencing this effect $[F(1,507)=4.97 \mathrm{p}<.05]$, as technical violation reincarcerations did not achieve statistical significance. This finding conflicts with best practice notions (Chandler, Fletcher, \& Volkow, 2009; Fletcher \& Chandler, 2006) and therefore needs further exploration. An examination into 
the relative redundancy of services in correctional interventions and their effect on behavior would be a useful starting point (see Kras, forthcoming; Shireman, 2010). 


\section{REFERENCES}

Andrews, D. A. \& Bonta, J. (2006). The psychology of criminal conduct (4 ${ }^{\text {th }}$ ed.). Newark, NJ: Anderson Publishing.

Andrews, D. A., Zinger, I., Hoge, R. D., Bonta, J., Gendreau, P., \& Cullen, F. T. (1990). Does correctional treatment work? A psychologically informed meta-analysis. Criminology, 28(3), 369-404.

Anglin, M. D. \& Hser, Y. I. (1990). Treatment of drug abuse. In M. Tonry \& J. Q. Wilson (Eds.), Drugs and Crime (pp. 393-340). Chicago, IL: University of Chicago Press.

Aos, S., Miller, M., \& Drake, E. (2006). Evidence-based adult corrections programs: What works and what does not. Olympia, WA: Washington State Institute for Public Policy.

Bahr, S. J., Masters, A. L., \& Taylor, B. M. (2012). What works in substance abuse treatment programs for offenders? The Prison Journal, 92(2), 155-174.

Bloom, D., Redcross, C., Zweig, J., \& Azurdia, G. (2007). Transitional jobs for exprisoners: Early impacts from a random assignment evaluation of the center for employment opportunities prisoner reentry program. New York, NY: Manpower Demonstration Research Corporation.

Blumstein, A. \& Beck, A. J. (1999). Population growth in U.S. prisons, 1980-1996. In M. Tonry \& J. Petersilia (Eds.), Prisons (pp. 17-61). Chicago, IL: University of Chicago Press.

Bouffard, J. A. \& Bergeron, L. E. (2007). Reentry works: The implementation and effectiveness of the Serious and Violent Offender Initiative. Journal of Offender Rehabilitation, 44(2/3), 1-29.

Bushway, S. D. \& Apel, A. (2012). A signaling perspective on employment-based reentry 
programming: Training completion as a desistance signal. Criminology and Public Policy, 11(1), 21-50.

Chandler, R. K., Fletcher, B. W., \& Volkow, N. D. (2009). Treating drug abuse and addiction in the criminal justice system: Improving public health and safety. Journal of the American Medical Academy, 301(2), 183-190.

Chanhatasilpa, C., MacKenzie, D., \& Hickman, L. (2000). The effectiveness of community-based programs for chemically dependent offenders: A review and assessment of research. Journal of Substance Abuse Treatment, 19(4), 383-393

Cohen, J. (1988). Statistical power for the behavioral sciences $\left(2^{\text {nd }}\right.$ ed.). Hillsdale, NJ: Lawrence Erlbaum Associates, Inc.

Cullen, F. T. \& Gendreau, P. (2000). Assessing correctional rehabilitation: Policy, practice, and prospects. In J. Horney (Ed.), Polices, Processes, and Decisions of the Criminal Justice System (pp. 109-175). Washington, DC: National Institute of Justice.

D’Agostino, R. B. (1971). A second look at analysis of variance on dichotomous data. Journal of Educational Measurement, 8(4), 327-333.

Dennis, M. L., Scott, C. K., Funk, R., \& Foss, M. A. (2005). The duration and correlates of addiction and treatment careers. Journal of Substance Abuse Treatment, 28(2), S51-S62.

Duwe, G. (2012). Evaluating the Minnesota comprehensive offender reentry plan (MCORP): Results from a randomized experiment. Justice Quarterly, 29(3), 347-383.

Erdfelder, E., Faul, F., \& Buchner, A. (1996). GPOWER: A general power analysis program. Behavior Research Methods, Instruments, and Computers, 28(1), 1-11.

Fletcher, B. \& Chandler, R. K. (2006). Principles of drug abuse treatment for criminal justice populations: A research-based guide. Bethesda, MD: US Department of Health and Human Services, National Institute of Health, National Institute on 
Drug Abuse.

Goldkamp, J. S. (2008). Missing the target and missing the point: 'Successful' random assignment but misleading results. Journal of Experimental Criminology, 4(2), 83-115.

Grommon, E. (2013). Prisoner reentry programs: Penetrating the black box for better theory and practice. El Paso, TX: LFB Scholarly Publishing.

Hiller, M. L., Knight, K., Saum, C. A., \& Simpson, D. D. (2006). Social functioning, treatment dropout, and recidivism of probationers mandated to a modified therapeutic community. Criminal Justice and Behavior, 33(6), 738-759.

Hiller, M. L., Knight, K., \& Simpson, D. D. (1999). Prison-based substance abuse treatment: Residential aftercare and recidivism. Addiction, 94(6), 833-842.

Hoffman, P. B. \& Beck, J. L. (1974). Parole decision-making: A salient factor score. Journal of Criminal Justice, 2(3), 195-206.

Hosmer, D., Lemeshow, S., \& May, S. (2008). Applied survival analysis: Regression modeling of time to event data ( $2^{\text {nd }}$ ed.). New York, NY: John Wiley and Sons.

Hser, Y., Anglin, M. D., Grella, C., Longshore, D., \& Prendergast, M. L. (1997). Drug treatment careers: A conceptual framework and existing research findings. Journal of Substance Abuse Treatment, 14(6), 543-558.

Jacobs, E. \& Western, B. (2007). Report on the evaluation of the comalert prisoner reentry program. Unpublished manuscript.

Kaplan, E. L. \& Meier, P. (1958). Nonparametric estimation from incomplete observations. American Statistical Association Journal, 53(282), 457-481.

Kras, K. R. (forthcoming). Offender perceptions of mandated substance abuse treatment: An exploratory analysis of offender experiences in a community-based treatment program. Journal of Drug Issues. 
Landenberger, N. A. \& Lipsey, M. (2006). The positive effects of cognitive-behavioral programs for offenders: A meta-analysis of factors associated with effective treatment. Journal of Experimental Criminology, 1(4), 432-450.

Lattimore, P. K., Barrick, K., Cowell, A., Dawes, D., Steffey, D., Tueller, S., \& Visher, C. A. (2012). Prisoner reentry services: What worked for SVORI evaluation participants? Washington, DC: National Instutite of Justice.

Lattimore, P. K. \& Visher, C. A. (2010). The multi-site evaluation of SVORI: Summary and synthesis. Research Triangle Park, NC: RTI International.

Lazowski, L. E. \& Miller, F. G., Boye, M. W., \& Miller, G. A. (1998). Efficacy of the substance abuse subtle screening inventory-3 (SASSI-3) in identifying substance dependence disorders in clinical settings. Journal of Personality Assessment, 71(1), 114128.

Lowenkamp, C. T., Latessa, E. J., \& Smith, P. (2006). Does correctional program quality really matter? The impact of adhering to the principles of effective intervention. Criminology and Public Policy, 5(2), 574-594.

Lunney, G. H. (1970). Using analysis of variance with dichotomous dependent variable: An empirical study. Journal of Educational Measurement, 7(4), 263-269.

MacKenzie, D. L. (2006). What works in corrections: Reducing the criminal activities of offenders and delinquents. New York, NY: Cambridge University Press.

Mallik-Kane, K. \& Visher, C. A. (2008). Health and prisoner reentry: How physical, mental, and substance abuse conditions shape the process of reintegration. Washington, DC: Urban Institute.

Maltz, M. (1984). Recidivism. Orlando, FL: Academic Press.

Marlowe, D. B. (2011). Evidence-based policies and practices for drug-involved offenders. The Prison Journal, 91(3 supplement), 27S-47S. 
Marlowe, D. B. (2006). When “what works” never did: Dodging the "scarlet M” in correctional rehabilitation. Criminology and Public Policy, 5(2), 339-346.

Marlowe, D. B. (2000). Coercive treatment of substance abusing criminal offenders. Journal of Forensic Psychology Practice, 1(1), 65-73.

McDonald, D., Dyous, C., \& Carlson, K. (2008). The effectiveness of prisoner reentry services as crime control: The Fortune Society. Cambridge, MA: Abt Associates.

Miller, F. G. \& Lazowski, L. E. (1999). The adult SASSI-3 manual. Springville, IN: The SASSI Institute.

Miller, W. R. \& Rollnick, S. (2002). Motivational interviewing: Preparing people for change ( $2^{\text {nd }}$ Ed.). New York, NY: Guilford Press.

Minnesota Department of Corrections. (2011). An evaluation of the prisoner reentry initiative:

Final report. St. Paul, MN: Minnesota Department of Corrections.

Mitchell, O., Wilson, D. B., \& MacKenzie, D. L. (2007). Does incarceration-based drug treatment reduce recidivism? A meta-analytic synthesis of the research. Journal of Experimental Criminology, 3(4), 353-375.

Morani, N. M., Wikoff, N., Linhorst, D. M., \& Bratton, S. (2011). A description of the selfidentified needs, service expenditures, and social outcomes of participants of a prisonerreentry program. The Prison Journal, 91(3), 347-365.

Mumola, C. J. \& Karberg, J. C. (2006). Drug use and dependence, state and federal prisoners, 2004. Washington, DC: US Department of Justice.

National Center on Addiction and Substance Abuse. (2010). Behind bars II: Substance abuse in America's prison population. New York, NY: National Center on Addiction and Substance Abuse at Columbia University.

Office of National Drug Control Policy. (2001). The national drug control strategy: 2001 annual report. Washington, DC: Office of National Drug Control Policy. 
Olson, D. E. \& Rozhon, J. (2011). A process and impact evaluation of the Sheridan correctional center therapeutic community program during fiscal years 2004 through 2010.Chicago, IL: Illinois Criminal Justice Information Authority.

Pearson, F. S. \& Lipton, D. S. (1999). A meta-analytic review of the effectiveness of corrections-based treatments for drug abuse. The Prison Journal, 79(4), 384-410.

Pelissier, B., Jones, N., \& Cadigan, T. (2007). Drug treatment aftercare in the criminal justice system: A systematic review. Journal of Substance Abuse Treatment, 32(3), 311-320.

Perry, A. E., Darwin, Z., Godrey, C., McDougall, C., Lunn, J., Glanville, J., \& Coulton, S. (2009). The effectiveness of interventions for drug-using offenders in the courts, secure establishments and the community: A systematic review. Substance Use and Misuse, 44(3), 374-400.

Prendergast, M. L. (2009). Interventions to promote successful re-entry among drug-abusing parolees. Addiction Science and Clinical Practice, 5(1), 4-13.

Prendergast, M. L., Farabee, D., Cartier, J., \& Henkin, S. (2002). Involuntary treatment within a prison setting: Impact on psychosocial change during treatment. Criminal Justice and Behavior, 29(1), 5-26.

Prendergast, M. L., Frisman, L., Sacks, J. Y., Stanton-Tindall, M., Greenwell, L., Lin, H., \& Cartier, J. (2011). A multi-site, randomized study of strengths-based case management with substance-abusing parolees. Journal of Experimental Criminology, 7(3), 225-253.

Prendergast, M., Greenwell, L., Cartier, J., Sacks, J., Frisman, L., Rodis, E., \& Havens, J. R. (2009). Adherence to scheduled sessions in a randomized field trial of case management: The criminal justice-drug abuse treatment studies transitional case management study. Journal of Experimental Criminology, 5(3), 273-297.

Prendergast, M. L., Podus, D., Chang, E., \& Urada, D. (2002). The effectiveness of drug abuse treatment: A meta-analysis of comparison group studies. Drug and Alcohol 
Dependence, 67(1), 53-72.

Redcross, C., Bloom, D., Azurdia, G., Zweig, J., \& Pindus, N. (2009). Transitional jobs for ex-prisoners: Implementation, two-year impacts, and costs of the center for employment opportunities prisoner reentry program. New York, NY: Manpower Demonstration Research Corporation.

Rossi, P. H., Lipsey, M. W., \& Freeman, H. E. (2004). Evaluation: A systematic approach $\left(7^{\text {th }}\right.$ ed.). Thousand Oaks, CA: Sage Publications.

Rossman, S. B. \& Roman, C. G. (2003). Case-managed reentry and employment: Lessons from the opportunity to succeed program. Justice Research and Policy, 5(2), 75-100.

Sample, L. L. \& Spohn, C. (2008). Final report for the evaluation of Nebraska's Serious and Violent Offender Reentry Program. Omaha, NE: University of Nebraska at Omaha, School of Criminology and Criminal Justice.

Seiter, R. P. \& Kadela, R. (2003). Prisoner reentry: What works, what does not, and what is promising. Crime and Delinquency, 49(3), 360-388.

Severson, M. E., Bruns, K., Veeh, C., \& Lee, J. (2011). Prisoner reentry programming: Who recidivates and when? Journal of Offender Rehabilitation, 50(6), 327-348.

Shadish, W.R., Cook, T.D., \& Campbell, D.T. (2002). Experimental and quasiexperimental designs for generalized causal inference. Boston, MA: Houghton Mifflin.

Shireman, R. M. (2010). Redundancy in a community corrections network: Testing the role of service-provider redundancy in Missouri's community correction implementation network. Unpublished master’s thesis. University of Missouri-Columbia, Columbia, Missouri.

Smith, L. G. \& Suttle, D. R. (2008). An outcome evaluation of the Pennsylvania 
Department of Corrections' Community Orientation and Reintegration Program. Columbus, OH: International Association of Reentry.

Stinchfield, R. \& Owen, P. (1998). Hazelden's model of treatment and its outcome. Addictive Behaviors, 23(5), 669-683.

Sung, H., Mahoney, A. M., \& Mellow, J. (2011). Substance abuse treatment gap among adult parolees: Prevalence, correlates, and barriers. Criminal Justice Review, 36(1), 40-57.

Tomlin, K. M. \& Richardson, H. (2004). Motivational interviewing and stages of change: Integrating best practices for substance abuse professionals. Center City, MN: Hazelden.

Travis, J. \& Visher, C. (2005). Viewing public safety through a reentry lens. In J. Travis and C. Visher (Eds.) Prisoner reentry and crime in America (pp. 1-14). New York, NY: Cambridge University Press.

Vaughn, T., Sarrazin, M. V., Saleh, S. S., Huber, D. L., \& Hall, J. A. (2002). Participation and retention in drug abuse treatment services research. Journal of Substance Abuse Treatment, 23(4), 387-397.

Vera Institute of Justice. (2010). The continuing fiscal crisis in corrections: Setting a new course. New York, NY: Vera Institute of Justice, Center on Sentencing and Corrections.

Wanberg, K. W., \& Milkman, H. B. (1998). Criminal conduct and substance abuse treatment: Strategies for self-improvement and change. Thousand Oaks, CA: Sage Publications.

Weisburd, D. (2000). Randomized experiments in criminal justice policy: Prospects and problems. Crime and Delinquency, 46(2), 181-193.

Wexler, H. K., Falkin, G. P., \& Lipton, D. S. (1990). Outcome evaluation of a prison therapeutic 
community for substance abuse treatment. Criminal Justice and Behavior, 17(1), 71-92.

Wilson, J. A. \& Davis, R. C. (2006). Good intentions meet hard realities: An evaluation of the project greenlight reentry program. Criminology and Public Policy, 5(2), 303-338.

Yamaguchi, K. (1991). Event history analysis. Newbury Park, CA: Sage Publications.

Zhang, S. X., Roberts, R. E. L., \& Callanan, V. J. (2006). Preventing parolees from returning to prison through community-based reintegration. Crime and Delinquency, 52(4), 551-571. 
Table 1. Demographic, Criminal History, and Substance Dependence Information

\begin{tabular}{|c|c|c|c|}
\hline & $\begin{array}{c}\text { Treatment } \\
(\mathrm{n}=263)\end{array}$ & $\begin{array}{l}\text { Control } \\
(\mathrm{n}=248)\end{array}$ & $\begin{array}{c}\text { Total } \\
(\mathrm{n}=511)\end{array}$ \\
\hline Age* & $35.57(9.00)$ & $33.73(8.92)$ & $34.68(9.00)$ \\
\hline Non-white & $70 \%$ & $63 \%$ & $67 \%$ \\
\hline \multicolumn{4}{|l|}{ Education } \\
\hline Less than High School & $39 \%$ & $36 \%$ & $38 \%$ \\
\hline GED & $51 \%$ & $47 \%$ & $49 \%$ \\
\hline More than High School* & $10 \%$ & $17 \%$ & $14 \%$ \\
\hline Previous Juvenile Commitment & $24 \%$ & $23 \%$ & \\
\hline Adult Probations & $1.44(1.44)$ & $1.38(1.27)$ & $1.41(1.36)$ \\
\hline Adult Jail & $3.33(4.00)$ & $2.85(3.81)$ & $3.10(3.91)$ \\
\hline Prison Admissions & $1.61(1.66)$ & $1.37(1.50)$ & $1.49(1.58)$ \\
\hline Previous Prison Admission & $68 \%$ & $65 \%$ & $67 \%$ \\
\hline \multicolumn{4}{|l|}{ Offense } \\
\hline Person & $41 \%$ & $39 \%$ & $40 \%$ \\
\hline Property & $25 \%$ & $25 \%$ & $25 \%$ \\
\hline Drugs & $34 \%$ & $31 \%$ & $32 \%$ \\
\hline Public Safety & $5 \%$ & $8 \%$ & $6 \%$ \\
\hline \multicolumn{4}{|l|}{ Assaultive Risk } \\
\hline High & $12 \%$ & $7 \%$ & $10 \%$ \\
\hline Medium & $52 \%$ & $53 \%$ & $53 \%$ \\
\hline Low & $36 \%$ & $40 \%$ & $37 \%$ \\
\hline \multicolumn{4}{|l|}{ Property Risk } \\
\hline High & $32 \%$ & $30 \%$ & $31 \%$ \\
\hline Medium & $59 \%$ & $61 \%$ & $60 \%$ \\
\hline Low & $9 \%$ & $9 \%$ & $9 \%$ \\
\hline \multicolumn{4}{|l|}{ Assessment of Drug Dependence } \\
\hline Low & $5 \%$ & $6 \%$ & $5 \%$ \\
\hline Moderate & $5 \%$ & $4 \%$ & $5 \%$ \\
\hline High & $54 \%$ & $56 \%$ & $55 \%$ \\
\hline Severe & $24 \%$ & $21 \%$ & $22 \%$ \\
\hline Random Pattern & $13 \%$ & $12 \%$ & $13 \%$ \\
\hline Treatment within Year of Release* & $20 \%$ & $29 \%$ & $24 \%$ \\
\hline
\end{tabular}


Table 2. Overall Fidelity of Program Implementation ( $\mathrm{n}=263)$

Phase One

Individual Counseling Sessions

Didactic Sessions

$.94(.18)$

$100 \%$

Family Group Sessions

$1.41(.33) \quad 100 \%$

$.60(.31) \quad 91 \%$

AA/NA Support Group Sessions

$1.85(.33)$

$98 \%$

Phase Two

Individual Counseling Sessions

$.27(.14)$

$91 \%$

Didactic Sessions

$.45(.21)$

$93 \%$

Family Group Sessions

$.18(.19)$

$11 \%$

AA/NA Support Group Sessions

$.45(.54)$

$39 \%$

Length of Stay (in days)

300 (112.79)

NOTE: Mean and standard deviation statistics for Phase One and Phase Two sessions are estimated by week. Proportion participated refers to the proportion of participants who attended at least one session across their length of stay in the program. 
Figure A. Kaplan-Meier Survival Curve of Relapse

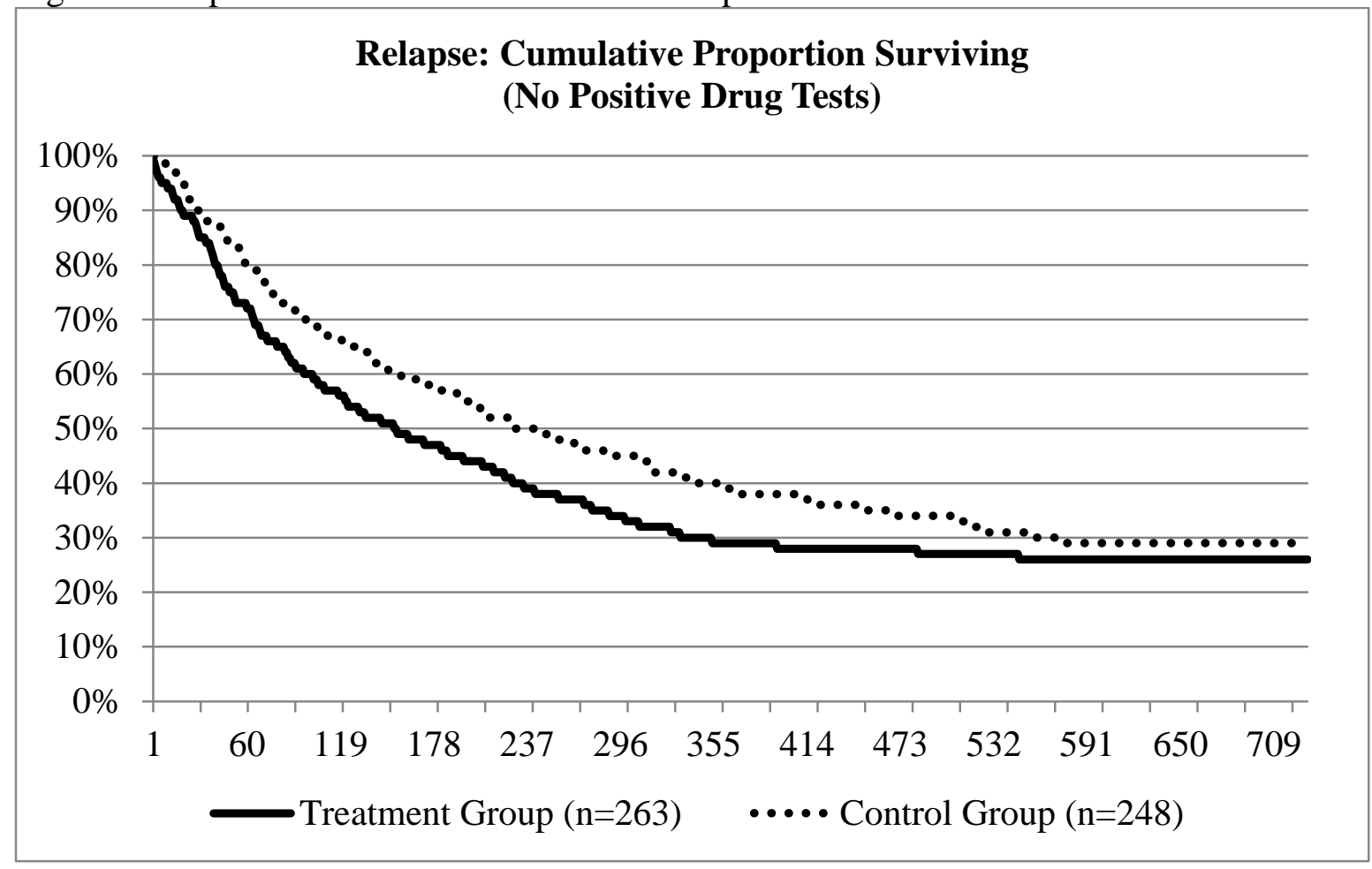


Table 3: Summary Table of Relapse and Recidivism Outcomes ( $\mathrm{n}=511)$

\begin{tabular}{|c|c|c|c|c|c|}
\hline & Mean & SE & $F$ & $\mathrm{df}$ & $\eta_{p}^{2}$ \\
\hline \multicolumn{6}{|c|}{ Relapse-At Least One Positive } \\
\hline Treatment & .75 & .03 & .64 & 1,509 & .001 \\
\hline Control & .71 & .03 & & & \\
\hline \multicolumn{6}{|c|}{ Relapse - Proportion Positive } \\
\hline Treatment & .26 & .02 & $4.46^{*}$ & 1,509 & .01 \\
\hline Control & 21 & .02 & & & \\
\hline \multicolumn{6}{|l|}{ Rearrest } \\
\hline Treatment & .30 & .03 & .74 & 1,509 & .001 \\
\hline Control & .27 & .03 & & & \\
\hline \multicolumn{6}{|c|}{ Reincarceration } \\
\hline Treatment & .36 & .03 & 1.49 & 1,509 & .003 \\
\hline Control & .31 & .03 & & & \\
\hline \multicolumn{6}{|c|}{ Technical Reincarceration } \\
\hline Treatment & .21 & .02 & 1.56 & 1,509 & .003 \\
\hline Control & .17 & .02 & & & \\
\hline \multicolumn{6}{|c|}{ New Sentence Reincarceration } \\
\hline Treatment & .14 & .02 & .06 & 1,509 & .000 \\
\hline \multirow[t]{2}{*}{ Control } & .14 & .02 & & & \\
\hline & Mean & SE & $\begin{array}{c}\text { Log Rank } \\
\text { Test Statistic }\end{array}$ & $\begin{array}{c}\text { Wilcoxon } \\
\text { Test Statistic }\end{array}$ & $\begin{array}{l}\text { Tarone-Ware } \\
\text { Test Statistic }\end{array}$ \\
\hline \multicolumn{6}{|c|}{ Relapse - At Least One Positive } \\
\hline Treatment & 286.02 & 17.50 & 3.56 & $6.43 * *$ & $5.18 *$ \\
\hline Control & 338.37 & 17.86 & & & \\
\hline \multicolumn{6}{|l|}{ Rearrest } \\
\hline Treatment & 602.58 & 13.50 & .87 & 1.02 & .95 \\
\hline Control & 623.87 & 12.66 & & & \\
\hline \multicolumn{6}{|c|}{ Reincarceration } \\
\hline Treatment & 604.34 & 12.21 & 1.90 & 2.35 & 2.12 \\
\hline Control & 633.73 & 10.58 & & & \\
\hline \multicolumn{6}{|c|}{ Technical Reincarceration } \\
\hline Treatment & 644.93 & 11.15 & 1.96 & 2.53 & 2.24 \\
\hline Control & 672.98 & 8.85 & & & \\
\hline \multicolumn{6}{|c|}{ New Sentence Reincarceration } \\
\hline Treatment & 680.58 & 8.30 & .24 & .24 & .24 \\
\hline Control & 684.67 & 7.96 & & & \\
\hline
\end{tabular}

${ }^{* *} \mathrm{p}<.01,{ }^{*} \mathrm{p}<.05$ 
Figure B. Kaplan-Meier Survival Curve of Recidivism

\section{Relapse: Cumulative Proportion Surviving}

(No Rearrests)
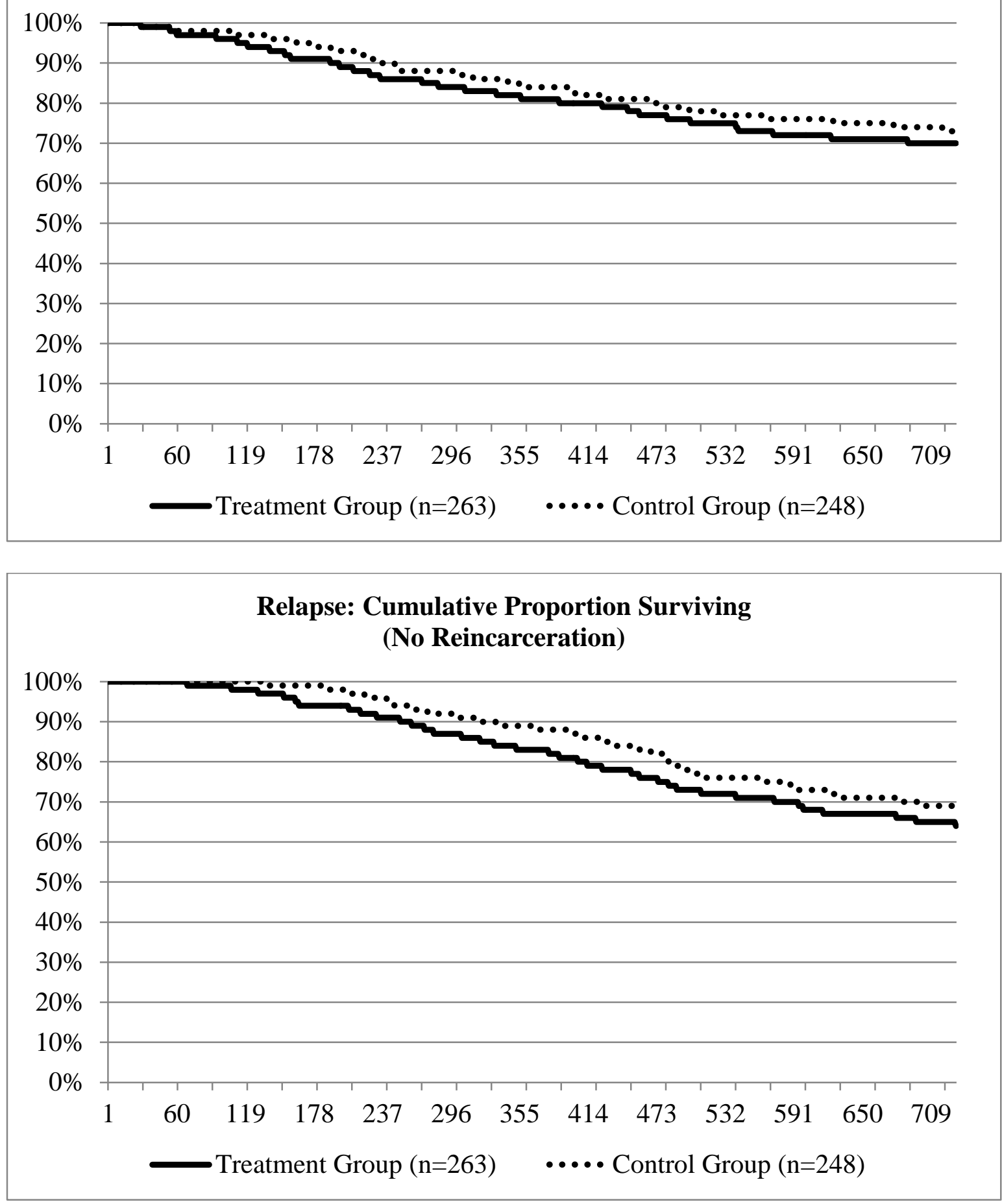